\title{
Cesarean delivery complicated with peripartum infection increases the risk of uterine rupture during subsequent trial of labour: A retrospective case-control study
}

\author{
Rina Tamir Yaniv ${ }^{1}$, Sivan Farladansky-Gershnabel ${ }^{2}$, Hadar Gluska $^{2}$, Yair Daykan ${ }^{3}$, Gil \\ Shechter Maor ${ }^{2}$, Ron Schonman ${ }^{4}$, and Tal Biron-Shental ${ }^{2}$ \\ ${ }^{1}$ Meir Medical Center, Kfar Saba, Israel, affiliated with the Sackler Faculty of Medicine, Tel \\ Aviv University, Tel Aviv \\ ${ }^{2}$ Meir Medical Center \\ ${ }^{3}$ Meir medical center \\ ${ }^{4}$ Meir Medical Centre
}

September 26, 2021

\begin{abstract}
Objective: To evaluate the relation between peripartum infection at first caesarean delivery to uterine dehiscence or rupture at the subsequent delivery. Design: Retrospective case-control study from March 2014 to October 2020. Setting: Universityaffiliated medical centre. Sample: Women with a prior caesarean delivery and proven dehiscence or uterine rupture diagnosed during their subsequent delivery. The control group included women who had a successful vaginal birth after Cesarean section without evidence of dehiscence or uterine rupture. Methods: We compared the rate of peripartum infection during the first Cesarean delivery and other relevant variables, between the two groups. We also analysed the type of infection correlated with uterine rupture or dehiscence. Main Outcome Measures: Rate of peripartum infection. Results: A total of 168 women were included, 71 with uterine rupture or dehiscence and 97 with successful vaginal birth after Cesarean section as the control group. The rate of peripartum infection at the first caesarean delivery was significantly higher in the study group compared to the control group ( $22.2 \%$ vs. $8.2 \%, \mathrm{p}=0.013)$. Multivariate logistic regression analysis found that peripartum infection remained an independent risk-factor for uterine rupture at the subsequent trial of labour after Cesarean delivery (95\% CI, P=0.018). We also found that endometritis had the highest correlation to uterine rupture $(9.8 \%$ vs. $0 \%, \mathrm{p}=0.02)$ Conclusion: Peripartum infection in the first caesarean delivery, may be an independent risk-factor for uterine rupture in a subsequent delivery. Compared to other infections, endometritis may pose the greatest risk for uterine rupture or dehiscence.
\end{abstract}

Cesarean delivery complicated with peripartum infection increases the risk of uterine rupture during subsequent trial of labour: A retrospective case-control study

Rina Tamir Yaniv MD, *Sivan Farladansky-Gershnabel MD, Hadar Gluska MD, Yair Daykan MD, Gil Shechter Maor MD, Ron Schonman MD, Tal Biron-Shental MD

Department of Obstetrics and Gynecology, Meir Medical Center, Kfar Saba, Israel, affiliated with the Sackler School of Medicine, Tel Aviv University, Israel

\section{Correspondence:}

Rina Tamir Yaniv

Department of Obstetrics and Gynecology 
Meir Medical Center

59 Tshernichovsky St.

Kfar Saba 44281 Israel.

Email: Rina.Tamir@clalit.org.il

Tel: 0097297472209

Fax: 0097297471262

Email: Rina.Tamir@clalit.org.il

Short title: Infection at $\mathrm{CD}$ and subsequent uterine rupture

Word count: 2173

Objective: To evaluate the relation between peripartum infection at first caesarean delivery to uterine dehiscence or rupture at the subsequent delivery.

Design: Retrospective case-control study from March 2014 to October 2020.

Setting: University-affiliated medical centre.

Sample: Women with a prior caesarean delivery and proven dehiscence or uterine rupture diagnosed during their subsequent delivery. The control group included women who had a successful vaginal birth after Cesarean section without evidence of dehiscence or uterine rupture.

Methods : We compared the rate of peripartum infection during the first Cesarean delivery and other relevant variables, between the two groups. We also analysed the type of infection correlated with uterine rupture or dehiscence.

Main Outcome Measures : Rate of peripartum infection.

Results : A total of 168 women were included, 71 with uterine rupture or dehiscence and 97 with successful vaginal birth after Cesarean section as the control group. The rate of peripartum infection at the first caesarean delivery was significantly higher in the study group compared to the control group $(22.2 \%$ vs. $8.2 \%$, $\mathrm{p}=0.013)$. Multivariate logistic regression analysis found that peripartum infection remained an independent risk-factor for uterine rupture at the subsequent trial of labour after Cesarean delivery (95\% CI, P=0.018). We also found that endometritis had the highest correlation to uterine rupture $(9.8 \%$ vs. $0 \%, \mathrm{p}=0.02)$

Conclusion : Peripartum infection in the first caesarean delivery, may be an independent risk-factor for uterine rupture in a subsequent delivery. Compared to other infections, endometritis may pose the greatest risk for uterine rupture or dehiscence.

Tweetable abstract: Peripartum infection in first caesarean delivery may be a risk-factor for subsequent uterine rupture

Key words: Cesarean section; dehiscence; endometritis; infection; postpartum fever; risk-factor; TOLAC; VBAC

Funding: This study was not funded.

\section{Introduction}

Cesarean delivery $(\mathrm{CD})$ is one of the most common surgeries worldwide, performed on one in four pregnant women in the UK (according to the NHS website) and up to four in five women in other regions of the world. ${ }^{1}$ The rate of subsequent vaginal birth after Cesarean section (VBAC) is low, and estimated to be $13.3 \%$ in the United States, ${ }^{2}$ and only 52.2 percent will attempt VBAC for their second birth in the UK. ${ }^{3}$ The implications of repeat $\mathrm{CD}$ on morbidity are profound and include higher rates of blood transfusions, surgical injuries and hysterectomies, with increased rates of complications as the number of CD increases. ${ }^{4,5}$ Therefore, many 
healthcare organizations try to increase VBAC rates. When consulting a woman regarding the mode of delivery, the risks and benefits of trial of labour after Cesarean (TOLAC) should be discussed, as well. ${ }^{6}$ Each woman should be advised according to her own obstetric and demographic characteristics, as well as her personal risk-factors for uterine rupture. ${ }^{7}$ Known risk-factors, such as previous arrest of descent, vertical uterine scar, etc. are well-established but infection of surgical site in the first CD is a possible risk-factor that has not been investigated in depth. The few studies that have been published are small and most did not isolate the type of infection as an independent risk factor. ${ }^{8}$ Currently, previous peripartum infections are not included into consultations regarding the preferred mode of delivery after a CD.

Peripartum infections may influence scar tissue healing and consequently affect uterine scar strength and ability to remain intact during subsequent pregnancies and deliveries. They include chorioamnionitis during labour, postpartum endometritis and other surgical site infections. Surgical site infection tends to occur within 30 days of the operation ${ }^{9}$ and may affect superficial or deep tissues. Pathogens commonly associated with obstetrical infections are Gram-negative bacilli, enterococci, Group B streptococci and anaerobes. ${ }^{10}$

It is well-established that surgical scar infection is a risk-factor for impaired healing. ${ }^{11}$ The pathogens interfere with leukocyte function and angiogenesis. Free radicals and cytotoxic enzymes are released from neutrophils, ${ }^{12,13}$ which may interfere with the healing process and leave the scar weaker and more vulnerable. Although it is not commonly considered a risk-factor for uterine rupture during labour, a surgical scar with previous surgical site infection (SSI) may have a higher tendency to rupture during a subsequent trial of labour.

The objective of this study was to investigate whether peripartum infection in the first CD is an independent risk-factor for uterine rupture in a subsequent delivery.

\section{Materials and Methods}

This retrospective case-control study was performed in a single academic medical centre from March 2014 to October 2020. Women with uterine rupture or dehiscence were included in the study group and compared to women who had a successful TOLAC (the control group). Demographic and obstetrical data, as well as any record of infection during or within a month after a prior CD were investigated.

Rupture of the uterus was defined as complete disruption of the previous Cesarean scar with communication between the uterine and abdominal cavities. Dehiscence of the scar was defined as incomplete disruption that included ruptured uterine muscle with intact serosa as the only layer keeping the foetus in the uterus. All cases of rupture and dehiscence were identified during CD.

The electronic medical records (EMR), were reviewed for demographic characteristics (age, BMI, gravity, and parity) and for medical information (regarding phase of delivery at first CD, surgical technique, and inter-delivery interval), and neonatal birth weight. Infection was defined as either intrapartum with maternal temperature above $38^{\circ} \mathrm{C}$ during or up to 24 hours after delivery. Postpartum fever was defined as maternal temperature above $38^{\circ} \mathrm{C}, 24-48$ hours after delivery, and SSI was defined according to the 2018 ACOG criteria and included endometritis, infected hematoma or infection of surgical wound up to 30 days from the previous CD. ${ }^{14}$ The diagnosis of SSI was determined based on the ICD-9 codes, analysis of laboratory data of wound cultures, non-prophylactic antibiotic prescriptions, and the clinical impression written by the medical team in the EMR.

Based on a uniform protocol, all patients undergoing CD received standard infection-prevention measures, including preoperative intravenous (IV) antibiotic prophylaxis with one to two grams of cefamezine based on maternal weight, surgical length and blood loss. For patients allergic to penicillin, clindamycin and gentamicin were administered. ${ }^{15}$

Intrapartum fever was treated with a combination of ampicillin and gentamicin, while patients with intrapartum fever undergoing CD also received IV metronidazole. Postpartum fever and endometritis were treated with IV Augmentin up to 48 hours from the last fever, according to protocols suggested by ACOG. ${ }^{16}$ Patients with large infected abdominal hematomas or any other severe infection were treated with a triad of 
ampicillin, gentamicin and IV metronidazole until improvement was noted or positive cultures allowed more specific treatment, according to institutional guidelines.

We compared three groups and subgroups: 1) All patients with rupture or dehiscence compared to controls (no rupture or dehiscence), 2) Rupture versus dehiscence, and 3) Rupture versus controls. We evaluated each group in terms of the type of infection as a risk-factor for rupture or dehiscence: intrapartum chorioamnionitis, postpartum endometritis, or SSI.

Data analysis

Statistical analysis was performed using SPSS, version 21.0 software (IBM Corp., Armonk, NY). Continuous variables were compared with Mann-Whitney U-test. Chi-square or Fisher's exact test was used for categorical data, as appropriate. Demographics and medical data were compared between the study and control group using univariate analysis. Potential confounders and significant variables in the univariate analysis were entered into multivariable binary regression analysis to establish their effect on the risk for uterine rupture. A probability value $<0.05$ was considered significant.

\section{Core Outcomes in Women's and Newborn Health (CROWN)}

A core outcome set (COS) was not used when designing this study because a relevant COS was not found in the CROWN database.

\section{Patient and Public Involvement}

Patients and the public were not involved in this retrospective study.

\section{Results}

A total of 71 women were included in the study group, of whom 40 experienced complete thickness uterine rupture and 31 had uterine scar dehiscence. They were compared to 97 cases of successful VBAC matched by maternal age, BMI, gestational age, and neonatal birth weights of the first and second deliveries.

The basic characteristics of the study and the control groups are shown in Table 1. Maternal age, gravidity and parity were higher in the study group, as expected by the study design. The study group included women with dehiscence or asymptomatic rupture in a repeat $\mathrm{CD}$, whereas the control group included only women after their first VBAC, which means they only had one prior CD. Although the study group was statistically older than the control group $(30.2 \pm 4.8$ years vs. $27.8 \pm 4.6$ years, $\mathrm{p}=0)$, this difference is not clinically significant. There were no significant differences between the groups regarding neonatal birth weight at first delivery and a statistical, but not clinically meaningful difference in birth weight at the second delivery. These potential confounders were included in the logistic regression (Tables 2,3). Infection in the previous delivery remained statistically significant, with odds ratio of 3.41

Sixteen of $71(22.5 \%)$ women with uterine rupture or dehiscence during the current birth, had an infection in proximity to their previous $\mathrm{CD}$, compared to 8 of $97(8.2 \%)$ in the control group $(\mathrm{p}=0.01)$.

We also compared the group with complete uterine rupture to the control group. Among 40 women with uterine rupture, $10(25 \%)$ had an infection at their first CD, as compared to 8 of $97(8.2 \%)$ of the controls $(\mathrm{p}=0.008)$. This result remained statistically significant in the logistic regression (Table 3$)$. There was no difference regarding the infection rate between the uterine rupture group and the dehiscence group ( $25 \%$ vs. $16 \%, \mathrm{p}=0.27)$.

We divided the type of infection into three groups: intrapartum fever, postpartum fever and SSI. SSI and in particular endometritis, had the strongest correlation to uterine rupture at subsequent delivery. None of the control group patients had endometritis, but 7 of $71(9.8 \%)$ in the study group and 5 of $40(12.8 \%)$ in the complete rupture group did. No significant difference was found between groups in terms of intrapartum fever (Table 4).

\section{Discussion}




\section{Main Findings}

This study found that women who experienced an infection at their previous CD had a significantly higher chance of uterine rupture at their next delivery. The type of infection found to be most correlated with uterine rupture was SSI, with endometritis being the most common type. The least correlated was chorioamnionitis (intrapartum fever), meaning that the timing of infection may have a decisive effect on the risk of rupture or dehiscence.

\section{Strengths and Limitations}

Limitations of this study are its retrospective nature and the small number of patients. Including dehiscence cases caused the groups to be heterogeneous in terms of gravity, parity and maternal age. However, none of these variables were significant in the regression analysis, showing that infection is well-correlated with uterine rupture. The average gestational age, was younger in the study group compared to the controls and birth weights were smaller. We believe this may be due to symptoms of rupture that developed earlier and led to an emergent CD. A COS was used, because there is no relevant core outcome in the database. Uterine rupture might be an important addition to the core outcomes.

\section{Interpretation}

Infection as a risk-factor for uterine rupture has not been studied in detail in the past. To the best of our knowledge, our cohort of 71 cases of uterine rupture is the largest to explore this subject, including 40 cases of complete rupture and 31 cases of dehiscence, as well as 97 controls.

Lieberman et al. ${ }^{7}$ found a correlation between infection and rupture. The rate of postpartum fever of any kind was $38 \%(8 / 21)$ in the uterine rupture group compared to $15 \%(13 / 84)$ in the control group ( $\mathrm{p}=0.03) .{ }^{8}$ Like our study, no statistical difference in the intrapartum fever group was found. They reviewed a database of patients from 1984 to 1996, with 70-75\% receiving antibiotic prophylaxis. Our results are based on deliveries from 2014 to 2022, with 100\% prophylaxis treatment according to ACOG guidelines and a very organized, prospective data collection system, based on ICD-9 codes and on detailed review of the EMR.

Gabbay-Benziv et al. found a correlation between complications at first CD (infection, post-partum haemorrhage) and uterine rupture during TOLAC $(\mathrm{p}=0.042) .{ }^{17}$ They had 3 cases of infection, all of which were postpartum fever documented during postpartum admission (4 days). In our study, 24 cases of infections were documented, 13 of which were diagnosed after readmission due to symptoms of infection.

Vilchez et al. used a large database of patients, and found that chorioamnionitis during the index delivery is a risk-factor for uterine rupture (odds ratio 5.7). ${ }^{18}$ They concluded that this was probably due to the association with protracted labour. Our study explored the impact of the infection itself on the uterine scar, evaluated at the next delivery.

\section{Conclusion}

Since our data come from a single tertiary centre, the treatment and surgical protocols were relatively uniform. Only $1 \%$ of the study group and $3 \%$ of the control group were treated with pitocin for augmentation during TOLAC $(\mathrm{p}=0.81)$. One patient in the control group was induced using an Atad catheter, as compared to none in the study group. All uterine closures of the first $\mathrm{CD}$ were done in two layers. The time interval between the CD and next delivery was 38.7+-24.4 months in the study group and 28.6+-10.7 months in the control group $(\mathrm{p}=0.00)$. However, there were large variations within the groups. It can be assumed that the experience of a traumatic labour (such as one accompanied by infection or re-hospitalization) may affect the time interval between deliveries but many other factors can affect this decision and this topic requires further research.

We analysed women with dehiscence or uterine rupture $(\mathrm{n}=71)$ and a subgroup of women with complete uterine rupture $(n=40)$. We did so because we believe that uterine rupture and dehiscence are one sequence of the same entity, with the same ethology. Thus, it is important to include cases of dehiscence in the analysis. On the other hand, we understand that including them may pose a confounder because some 
patients in the control group may have had a subclinical dehiscence during their VBAC, and since we do not perform a routine scar test after successful VBAC, these cases could be underdiagnosed. Therefore, we chose to present the groups together and separately and show that in both, infection in the first delivery was found to be a risk-factor for uterine rupture.

This study investigated a possible new risk-factor for a disastrous event. Further studies are needed to establish this finding but this is a manageable risk and its incidence can be reduced.

Knowing that infection in general and endometritis specifically may increase the risk for uterine rupture in the next delivery, should inform the treating staff on the following issues: when consulting with a patient on repeat $\mathrm{CD}$ versus TOLAC, endometritis after the previous $\mathrm{CD}$ should be included among the considerations, along with other traditional risk-factors. In addition, our results emphasize the importance of adhering to the guidelines for perioperative preventative treatment of infection, and finally, diagnosed infection in general and endometritis, in particular, should be treated quickly and accurately.

\section{Acknowledgments}

Disclosure statement: The authors report no conflict of interests.

\section{Contribution to Authorship}

Conceptualization: Rina Tamir Yaniv. Methodology: Sivan Farladansky-Gershnabel Formal Analysis: Sivan Farladansky-Gershnabel. Investigation: Hadar Gluska, Resources: Hadar Gluska. Data Curation: Gil Shechter Maor. Writing - Original Draft Preparation: Rina Tamir Yaniv. Writing - Review \& Editing: Ron Schonman, Tal Biron-Shental. Visualization: Gil Shechter Maor. Supervision: Tal Biron-Shental. Project Administration: Rina Tamir Yaniv

Ethics approval: The Meir Medical Center Institutional Review Board approved the study, which was performed in accordance with the Ethical Standards of the Declaration of Helsinki and its later amendments, on 22 October 2020, approval number MMC-0239-20. Informed consent was waived due to the retrospective design of the study.

Funding: This work was not funded.

Data sharing: Data will be available upon request

\section{References}

1. Antoine C, Young BK, Young B. Cesarean section one hundred years 1920-2020: the Good, the Bad and the Ugly. J Perinat Med [Internet]. 2020 Sep 4 [cited 2021 Apr 2];49(1):5-16. Available from: http://www.ncbi.nlm.nih.gov/pubmed/32887190

2. Osterman MJK. Recent trends in vaginal birth after Cesarean delivery: United States, 2016-2018. NCHS Data Brief. 2020 Mar;(359):1-8.

3. Knight HE, Gurol-Urganci I, Van Der Meulen JH, Mahmood TA, Richmond DH, Dougall A, et al. Vaginal birth after caesarean section: A cohort study investigating factors associated with its uptake and success. BJOG. 2014;121(2):183-92.

4. Cook JR, Jarvis S, Knight M, Dhanjal MK. Multiple repeat caesarean section in the UK: Incidence and consequences to mother and child. A national, prospective, cohort study. BJOG. 2013; 120:772. doi: $10.1111 / 1471-0528.12158$.

5. Marshall NE, Fu R, Guise JM. Impact of multiple cesarean deliveries on maternal morbidity: a systematic review. Am J Obstet Gynecol. 2011;205:262.e1-8.

doi: 10.1016/j.ajog.2011.06.035.

6. Joseph KS, Shiliang L, Muraca GM, Sabr Y, Pressey T, Liston RM. Mode of delivery after a previous cesarean birth, and associated maternal and neonatal morbidity. Cmaj. 2018;190(18):E556-64. 
7. Grobman WA, Lai Y, Landon MB, Spong CY, Leveno KJ, Rouse DJ, et al. Development of a nomogram for prediction of vaginal birth after cesarean delivery. Obstet Gynecol. 2007;109(4):806-12.

8. Shipp TD, Zelop C, Cohen A, Repke JT, Lieberman E. Post-Cesarean delivery fever and uterine rupture in a subsequent trial of labor. Obstet Gynecol. 2003;101:136-9. doi: 10.1016/s0029-7844(02)02319-0

9. Gur R, Duggal SD, Rongpharpi SR, Srivastava R, Kumar A, Gupta V, et al. Post caesarean surgical site infections. Arch Clin Microbiol. 2015;6:4

10. Owens CD, Stoessel K. Surgical site infections: epidemiology, microbiology and prevention. J Hosp Infect. 2008; 70 Suppl 2:3-10. doi: 10.1016/S0195-6701(08)60017-1.

11. Wolcott RD, Rhoads DD, Dowd SE. Biofilms and chronic wound inflammation. J Wound Care. 2008 17(8):333-41. doi: 10.12968/jowc.2008.17.8.30796.

12. Davis SC, Ricotti C, Cazzaniga A, Welsh E, Eaglstein WH, Mertz PM. Microscopic and physiologic evidence for biofilm-associated wound colonization in vivo. Wound Repair Regen. 2008; 16(1):23-9. doi: 10.1111/j.1524-475X.2007.00303.x

13. Black CE, Costerton JW. Current concepts regarding the effect of wound microbial ecology and biofilms on wound healing. Surg Clin North Am. 2010;90:1147-60. doi: 10.1016/j.suc.2010.08.009.

14. Infections P. ACOG Practice Bulletin No. 195 Summary: Prevention of Infection After Gynecologic Procedures. Obstet Gynecol. 2018;131(6):1177-9.

15. Antibiotics P. ACOG Practice Bulletin No. 199: Use of prophylactic antibiotics in labor and delivery. Obstet Gynecol. 2018;132(3):e103-19.

16. Gynecologists $\mathrm{T}$ american college of obstetricians and. Intrapartum Management of Intraamniotic Infection. Obstet Gynecol. 2017;130(2):e95-101.

17. Salman L, Hiersch L, Shmueli A, et al. Complicated primary cesarean delivery increases the risk for uterine rupture at subsequent trial of labor after cesarean. Arch Gynecol Obstet. 2018;298(2):273-7. Available from: https://doi.org/10.1007/s00404-018-4801-x

18. Vilchez Gustavo, Nazeer S, Kumar K, Warren M, Dai J, Sokol RJ. Contemporary epidemiology and novel predictors of uterine rupture: a nationwide population-based study. Arch Gynecol Obstet. 2017;296:869-75.

\section{Hosted file}

Tables 12.7.21.docx available at https://authorea.com/users/437886/articles/539234-cesareandelivery-complicated-with-peripartum-infection-increases-the-risk-of-uterine-ruptureduring-subsequent-trial-of-labour-a-retrospective-case-control-study 